\title{
High SMARCA5 Expression is Associated With Poor Prognosis in Patients With Non-Small Cell Lung Cancer
}

\section{Zhi-Qiang Yang}

Zhoushan Hospital

yuanyuan wen ( $\nabla$ wenyuanyuan1022@sina.cn )

Zhoushan Hospital

Hai-Feng Li

Zhoushan Hospital

Lue Li

Zhoushan Hospital

Hui He

Zhoushan Hospital

\section{Research}

Keywords: SMARCA5, Prognosis, Proliferation, Invasion, NSCLC

Posted Date: June 17th, 2020

DOI: https://doi.org/10.21203/rs.3.rs-35749/v1

License: (c) (1) This work is licensed under a Creative Commons Attribution 4.0 International License. Read Full License 


\section{Abstract}

Background: SMARCA5 has been proved to play the role of oncogene in various types of tumors. The purpose of our study was to study the relationship between the expression of SMARCA5 and the prognosis of patients with Non-small cell lung cancer(NSCLC), and to investigate the effect of silencing the expression of SMARCA5 on the biological behavior of NSCLC.

Methods $\ \mid$ mmunohistochemistry method was adopted to detect to the expressions of SMARCA5 proteins in NSCLC and normal lung tissues. The correlation between the expression of SMARCA5 and clinicopathological factors and survival prognosis of patients with NSCLC were analyzed by statistical methods. Western blot and RT-PCR were used to detect the expression of SMARCA5 in normal human bronchial epithelial cell line (HBE) and human lung cancer cell lines (A549, Lu165, SK-MES-1 and NCIH520). SMARCA5 siRNA was employed to silence the the expression of SMARCA5. MTT and Transwell methods were used to detect the proliferation and invasion of lung cancer cells.

Results: SMARCA5 protein were highly expressed in 78 (59.5\%) NSCLC tissues. SMARCA5 mRNA $(P<0.05)$ and protein $(P<0.05)$ were significantly higher in NSCLC tissues than in the adjacent normal tissues. SMARCA5 expression was significantly associated with poorly differentiation $(P<0.001)$, advanced TNM stage $(P<0.001)$, and pleural invasion $(P=0.032)$. Meanwhile, High expression of SMARCA5 was associated with poor prognosis of NSCLC patients. Knockdown of SMARCA5 expression inhibited cell growth and invasion in NSCLC.

Conclusion: High expression of SMARCA5 is associated with poor prognosis in patients with NSCLC, and SMARCA5 can increase activity of proliferation and invasion in NSCLC cells. SMARCA5 may play a decisive role in tumorigenesis of NSCLC.

\section{Background}

Lung cancer is a common malignant tumor in the world and the leading cause of death of cancer patients (1). Non-small cell lung cancer (NSCLC) is the primary histological of lung cancer. Many treatments, including surgery, radiation therapy and chemotherapy, have been used in lung cancer patients, but the prognosis is poor (2). Therefore, new molecular markers are urgently needed for early detection and prognostic analysis.

The SWI/SNF (SWItch/sucrose nonfermentable) is a complex nucleosomal transformation that has been found in both eukaryotes and prokaryotes, and is associated with transcriptional control of gene expression in the ATP-dependent manner, which plays an important role in DNA repair and replication, cell growth, and cell differentiation(3-5). SMARCA5 also known as human sucrose nonfermenting protein 2 homologue(hSNF2H ), is one of the SWI/SNF family proteins that has the activity of helicase and ATPase (6-10). SMARCA5 controls the sensitivity of DNA through histone octamers, which is of great significance for gene expression, DNA replication, DNA repair and maintenance of higher-order chromatin structures $(6,11-18)$. SMARCA5 is an oncogene in various types of tumors. Studies have shown that 
SNF2H can promote the growth and proliferation of liver cancer cells(19). At the same time, SMARCA5 is associated with poor prognosis of breast cancer patients and can promote the proliferation and invasion of breast cancer cells(20). Other studies have observed that the overexpression of SMARCA5 can promote the proliferation of gastric cancer, which play an important role in gastric carcinogenesis(21). But its biological functions and expression pattern in NSCLC is unintelligible. The biological functions of SMARCA5 in the development and progression of NSCLC were explored by examining the expression of SMARCA5 in NSCLC tissues and cell lines in the present study. our study revealed that SMARCA5 expression is upregulated in NSCLC and is significantly associated with progression and poor prognosis in NSCLC patients. Furthermore, we found that knockdown of SMARCA5 inhibited the invasion abilities and cell proliferation of NSCLC cells.

\section{Methods}

Tissue samples and patients

We collected 131 lung tissue samples from zhoushan hospital from January 1, 2007 to December 31, 2016. All enrolled patients underwent radical surgical resection without chemotherapy or radiotherapy. Fresh tissue specimens were fixed in formalin and embedded in paraffin for tissue sectioning. Hematoxylin and eosin staining (H\&E) were used for routine staining. The histological type was determined by two senior pathologists according to the World Health Organization lung carcinoma histological classification criteria (2015). According to TNM staging system of the International Union Against Cancer (UICC) in 2009 Informed consent was obtained prior to surgery from all enrolled patients. The follow-up methods were outpatient and telephone. The survival time was measured from the date of surgery to the date of death due to recurrence/metastasis or the date of the last follow-up. The deadline of follow-up data in this paper was December 2016, and one patient lost the follow-up.

Immunohistochemical staining and results determination

The tissue was fixed in neutral formalin and embedded in paraffin, and $4 \mu \mathrm{m}$ sections were prepared. Immunostaining was performed by the streptavidin-peroxidase (S-P) method (UltrasensitiveTM MaiXin, Fuzhou, China). The primary antibodies were polyclonal mouse anti- SMARCA5 antibody ( hSNF2H, sc365727 1:600; Santa Cruz Biotechnology, Inc., Santa Cruz, CA). The peroxidase reaction was developed with 3, 3'-diaminobenzidine tetrahydrochloride (DAB, MaiXin, Fuzhou, China). Nonimmune serum was used as the negative control instead of the primary antibody. All the samples were evaluated by two independent pathologists. The presence of brown-yellow granules in the nucleus/cytoplasm was considered as positive staining. The intensity of immunostaining ( 0 , negative; 1 , moderate; and 2 , strong) and the staining percentage of tumor cells $(0,0 \% ; 1,1-5 \% ; 2,6-25 \% ; 3,26-75 \%$; and $4,76-100 \%)$ were assessed in at least 5 high power fields ( $400 \times$ magnification). The scores of each tumor sample were multiplied to give a final score of 0 to 8 , and the tissues with a final score of 4 -8were finally determined as SMARCA5 overexpression(20).

Cell Culture 
The normal human bronchial epithelial cell line (HBE),human lung adenocarcinoma cell lines A549 and Lu165ロhuman squamous cell carcinoma cell lines SK-MES-1 and NCI-H520 were maintained in RPMI 1640 medium (GIBCO, Inc., Grand Island, NY, USA) supplemented with 10\% fetal bovine serum(GIBCO, Inc., Grand Island, NY, USA) and $100 \mathrm{U} / \mathrm{mL}$ of penicillin-streptomycin in a humidified atmosphere with $5 \%$ of $\mathrm{CO}_{2}$.

Small RNA Interference

Cells were inoculated with a six-well plate at a density of $5 \times 10^{5}$ per well. The untreated group and the control siRNA group were used as negative controls, siRNA concentrations was 20 um. $10 \mu$ SMARCA5 siRNA (hSNF2H siRNA, sc-35594, Santa Cruz Biotechnology, Inc., Santa Cruz, CA) and 10 ug control siRNA were mixed with $1.5 \mathrm{~mL}$ double free culture medium at room temperature respectively. The untreated group was cultured in normal medium. 60 $\mu \mathrm{L}$ Lipofectamine 2000 was mixed with $1.5 \mathrm{~mL}$ double free culture medium and incubated at room temperature for $5 \mathrm{~min}$. The diluted siRNA was mixed with Lipofectamine2000 and incubated at room temperature for $30 \mathrm{~min}$. A549 and SK-MES-1 cells were transfected with the mixture. After $6 \mathrm{~h}$, the cells were transferred to normal medium and cultured for $36 \mathrm{~h}$ after transfection. Then cells were collected for studying. The experiments were divided into SMARCA5 siRNA group, control siRNA group and untreated group.

Western blot analysis

Samples of $1 \sim 2 \mathrm{~g}$ were taken, and lysis buffer of about 5 times wet weight was added. After homogenization was crushed, or cells were collected and washed with pre-cooled PBS for 3 times. $500 \mu \mathrm{L}$ cell lysis fluid and protease inhibitor complex were added and treated with ultrasonic treatment under ice bath. The supernatants were centrifuged at $10000 \mathrm{~g}$ for $30 \mathrm{~min}$ at $4{ }^{\circ} \mathrm{C}$. The supernatants containing total protein were harvested. Aliquots containing $60 \mu \mathrm{g}$ of proteins were separated on a $12 \%$ SDSpolyacrylamide gel and transferred to polyvinylidene fluoride membranes and incubated overnight at $4{ }^{\circ} \mathrm{C}$ with primary antibody against SMARCA5 (1:1000) and $\beta$-actin(1:200), and incubated overnight at $4^{\circ} \mathrm{C}$. Then added into the secondary antibody (1:2000), incubated at room temperature for $2 \mathrm{~h}$. Then the results were visualized by ECL (Pierce Biotechnology, Rockford, IL, USA). Images were collected by the gel imaging system, The grayscale values of the SMARCA5 bands were normalized to the corresponding $\beta$ actin band to determine the expression level of the protein.

RT-PCR analysis

Total RNA was isolated using Trizol reagent (Invitrogen) according to the manufacturer's instructions. cDNA was obtained by reverse transcription using the RNA PCR Kit (AMV), and SMARCA5 was amplified. $\beta$-actin was used as an internal parameter. The conditions for synthesizing cDNA were: $30^{\circ} \mathrm{C}$ for $10 \mathrm{~min}$, $42{ }^{\circ} \mathrm{C}$ for $30 \mathrm{~min}, 99^{\circ} \mathrm{C}$ for $5 \mathrm{~min}$ and $5^{\circ} \mathrm{C}$ for $5 \mathrm{~min}$.

MRNA was amplified using reverse transcriptional cDNA as a template. The primers used in this study were: SMARCA5: forward, 5 '- CCTTTG AAG ATG AAA CCA GGG CGC-3', and reverse, 5 '- CTG 
TTAATAGCTCTTCATCCTCCTC-3'; and $\beta$-actin: forward, 5'-AAATCGTGCGTGACATTAA-3', and reverse, 5'CTCGTC ATACTCCTGCTTG - 3'. The PCR products were stained with ethidium bromide and analyzed using a Bioimaging system (UVP) after conducting electrophoresis. Relative band intensities were determined using $\mathrm{NIH}$ image software.

Matrigel invasive assays.

Matrigel (BD Biosciences) and Transwell inserts of $8.0 \mathrm{~mm}$ pore size (Corning, Inc., NY, USA) were used for invasion assays. The Matrigel $(\mu \mathrm{g} / \mathrm{ml})$ was applied to the upper surface of the membranes. After transfection for $24 \mathrm{~h}$, cells were seeded on the upper chamber $\left(5 \times 10^{4}\right.$ cells / well) and incubated for $24 \mathrm{~h}$. Cells that had invaded the surface of the membrane were fixed with methanol and stained with hematoxylin. Five random high-magnification microscope fields per filter were counted. The experiment was repeated thrice and the data were shown in meanstandard deviation (SD), the experiments were divided into SMARCA5 siRNA group, control siRNA group and untreated group.

\section{MTT assay}

The transfected cells were seeded in 96 -well plates $\left(1 \times 10^{4}\right.$ cells $/$ well $)$. Cell proliferation was evaluated each day for 4 days after transfection using MTT method. The absorbance, which was directly proportional to the number of living cells in culture, was measured at $570 \mathrm{~nm}$ using a microplate reader (Model 550; Bio-Rad, Hercules, CA, USA). A blank with dimethyl sulfoxide alone was measured and subtracted from all values. All the assays were done at least three times independently.

\section{Statistical analysis}

SPSS version 18.0 for Windows was used for all analyses. The Pearson Chi-Square test was used to examine the correlation between the expression of SMARCA5 and clinicopathological factors. The Student's t-test was used to compare data from the densitometry analysis of western blotting and RTPCR. The Kaplan-Meier method was used to estimate the probability of patient survival. The Cox's proportional hazard regression model was used to estimate the possible prognostic significance of clinicopathological variables. $P$ values less than 0.05 were considered statistically significant.

\section{Results}

1. The high expression of SMARCA5 was associated with high pTNM stage, poor differentiation and Pleural invasion in NSCLC

To determine the expression of SMARCA5 protein in lung cancer tissues, we examined the expression of SMARCA5 protein in 131 lung cancer tissues by immunohistochemical method. It was located in the nucleus of the tumor cell, which was not expressed in normal bronchial epithelium or alveolar epithelium(Fig. 1a, b), showed strong positive nuclear staining in 78 (59.5\%) lung cancer tissues(final 
score: $\geq 4$ points)(Fig. 1C, e), and weak positive expression in 53 cases (40.5\%)(final score: $<4$ points) (Fig. 1d, f).

To evaluate the role of SMARCA5 protein in the malignant development of NSCLC, we analyzed the relationship between SMARCA5 protein expression and the histopathological stage of NSCLC. We found that high SMARCA5 expression was significantly associated with poorly differentiation $(P=0.000)$, advanced TNM stage $(P=0.000)$, and pleural invasion $(P=0.032)$ (Table 1$)$. The expression of SMARCA5 was independent of age, sex, histology, tumor size, tumor location, and lymph nodes (Table 1). 
Table 1

Expression of SMARCA5 in 131 NSCLC and its correlation with clinicopathological parameters

\begin{tabular}{|c|c|c|c|c|c|c|}
\hline \multirow[t]{2}{*}{ Characteristics } & & \multirow[t]{2}{*}{$\mathrm{N}$} & \multicolumn{2}{|l|}{ SMARCA5 } & \multirow[t]{2}{*}{$x^{2}$} & \multirow[t]{2}{*}{$P$} \\
\hline & & & $\begin{array}{l}\text { Low } \\
\text { expression }\end{array}$ & $\begin{array}{l}\text { High } \\
\text { expression }\end{array}$ & & \\
\hline \multirow[t]{2}{*}{ Gender } & male & 73 & 27 & 46 & 0.825 & 0.364 \\
\hline & female & 58 & 26 & 32 & & \\
\hline \multirow[t]{2}{*}{ Age(year) } & $\geq 60$ & 80 & 30 & 50 & 0.746 & 0.388 \\
\hline & $<60$ & 51 & 23 & 28 & & \\
\hline \multirow[t]{2}{*}{ Histological } & $\operatorname{scc}$ & 47 & 16 & 31 & 1.252 & 0.263 \\
\hline & adenocarcinoma & 84 & 37 & 47 & & \\
\hline \multirow[t]{3}{*}{ Tumor Size(cm) } & $\leq 2$ & 46 & 24 & 22 & 4.942 & 0.085 \\
\hline & $2-3$ & 32 & 13 & 19 & & \\
\hline & $\geq 3$ & 53 & 16 & 37 & & \\
\hline \multirow[t]{2}{*}{ Tumor Location } & left lung & 52 & 16 & 36 & 3.36 & 0.067 \\
\hline & right lung & 79 & 37 & 42 & & \\
\hline \multirow{2}{*}{$\begin{array}{l}\text { Lymph Node } \\
\text { Status }\end{array}$} & + & 39 & 11 & 28 & 3.461 & 0.063 \\
\hline & - & 92 & 42 & 50 & & \\
\hline \multirow[t]{2}{*}{ Pleural Invasion } & + & 41 & 11 & 30 & 4.601 & 0.032 \\
\hline & - & 90 & 42 & 48 & & \\
\hline \multirow[t]{3}{*}{ Differentiation } & well & 52 & 32 & 20 & 16.666 & 0.000 \\
\hline & moderate & 41 & 11 & 30 & & \\
\hline & poor & 38 & 10 & 28 & & \\
\hline \multirow[t]{4}{*}{ Clinical Staging } & 0 & 11 & 10 & 1 & & \\
\hline & ૫ & 58 & 30 & 28 & 24.462 & \\
\hline & प & 53 & 11 & 42 & & 0.000 \\
\hline & ૧ & 9 & 2 & 7 & & \\
\hline
\end{tabular}

We also examined the SMARCA5 protein in 40 fresh lung cancer tissues and paired adjacent normal tissues. RT-PCR and Western blot results showed that the level of SMARCA5 mRNA $(P<0.05)$ and protein 
$(P<0.05)$ were significantly higher in lung cancer tissues than in the adjacent normal tissues, respectively(Fig. 2).

2. High expression of SMARCA5 was associated with poor prognosis of NSCLC patients.

A survival curve analysis was adopted for patients with complete postoperative follow-up records. In the 131 NSCLC, the overall survival rate of patients with high expression of SMARCA5 was significantly lower than that of patients with low expression SMARCA5 (Log-Rank test, $P=0.001$ ) (Fig. 3). Univariate analysis showed that SMARCA5 expression status $(P=0.001)$, tumor size $(P=0.003)$, differentiation $(P=$ $0.001)$, lymph node status $(P=0.000)$ and TNM stage $(P=0.000)$ were closely related to the prognosis of patients (Table 2). Multivariate survival analysis showed that SMARCA5 expression (Risk ratio: 2.846, $P=$ 0.012 ), differentiation (Risk ratio: $3.202, P=0.000$ ), TNM stage(Risk ratio: $2.146, P=0.005$ ) and lymph node status (Risk ratio: 3.358, $P=0.000$ ) were independent risk factors for lung cancer prognosis (Table 3). 
Table 2

Univariate analysis of overall survival rate in 131 patients with NSCLC

\begin{tabular}{|c|c|c|c|c|}
\hline Characteristics & & $\mathbf{N}$ & $\begin{array}{l}\text { Chi-Square } \\
\left(x^{2}\right)\end{array}$ & $\begin{array}{l}\text { Log Rank Test } \\
(P)\end{array}$ \\
\hline \multirow[t]{2}{*}{ SMARCA5 Expression } & low & 53 & 24.013 & 0.001 \\
\hline & high & 78 & & \\
\hline \multirow[t]{2}{*}{ Gender } & male & 73 & 1.491 & 0.222 \\
\hline & female & 58 & & \\
\hline \multirow[t]{2}{*}{ Age (years) } & $\geq 60$ & 80 & 0.168 & 0.682 \\
\hline & $<60$ & 51 & & \\
\hline \multirow[t]{2}{*}{ Histology } & $\operatorname{scc}$ & 47 & 0.237 & 0.627 \\
\hline & adenocarcinoma & 84 & & \\
\hline \multirow{3}{*}{$\begin{array}{l}\text { Tumor Size } \\
(\mathrm{cm})\end{array}$} & $\leq 2$ & 46 & 11.408 & 0.003 \\
\hline & $2-3$ & 32 & & \\
\hline & $\geq 3$ & 53 & & \\
\hline \multirow[t]{2}{*}{ Tumor Location } & left lung & 52 & 0.338 & 0.561 \\
\hline & right lung & 79 & & \\
\hline \multirow[t]{2}{*}{ Lymph Node Status } & + & 39 & 26.268 & 0.000 \\
\hline & - & 92 & & \\
\hline \multirow[t]{2}{*}{ Pleural Invasion } & + & 41 & 0.09 & 0.764 \\
\hline & - & 90 & & \\
\hline \multirow[t]{3}{*}{ Differentiation } & well & 52 & 36.286 & 0.001 \\
\hline & moderate & 41 & & \\
\hline & poor & 38 & & \\
\hline \multirow[t]{4}{*}{ Clinical Staging } & 0 & 11 & & \\
\hline & Q & 58 & & \\
\hline & 口 & 53 & 65.371 & 0.000 \\
\hline & प & 9 & & \\
\hline
\end{tabular}


Table 3

Multivariate analysis for predictive factors in 131 patients with NSCLC (Cox regression model)

\begin{tabular}{|llll|}
\hline Factor & Risk ratio & $\mathbf{9 5 \% c o n f i d e n c e ~ i n t e r v a l ~}$ & $\boldsymbol{P}$ \\
\hline SMARCA5 Expression & 2.846 & $1.263-6.411$ & 0.012 \\
\hline Tumor Size & 1.279 & $0.803-2.037$ & 0.331 \\
\hline Lymph Node Status & 3.358 & $1.725-6.538$ & 0.000 \\
\hline Differentiation & 3.202 & $2.109-4.860$ & 0.000 \\
\hline TNM Stage & 2.146 & $1.253-3.676$ & 0.005 \\
\hline
\end{tabular}

3. Silencing SMARCA5 could down-regulate SMARCA5 expression in NSCLC

We examined the expression of SMARCA5 in four lung cancer cell lines(A549, Lu165, SK-MES-1 and NClH520) and normal bronchial epithelial cell line (HBE) by western blot and RT-PCR. We found that the expression of SMARCA5 in HBE cell lines $(P<0.05)$ was lower than that in lung cancer cell lines (Lu165, NCI-H520, A549, SK-MES-1). In the four lung cancer cell lines, the expression of SMARCA5 was higher in A549 $(P<0.05)$ and SK-MES-1 $(P<0.05)$ than in NCl-H520 and Lu165 (Fig. 4).

We adopted SMARCA5 siRNA in A549 and SK-MES-1 cell lines to knockdown SMARCA5 expression. After SMARCA5 siRNA fragment was imported into A549 and SK-MES-1 cells for $36 \mathrm{~h}$, RT-PCR and western blot results showed that, compared with untreated group or control group, the expression of SMARCA5 mRNA $(P<0.05)$ and protein $(P<0.05)$ in both lung cancer cells were significantly down-regulated, which was statistically significant(Fig. 5). These results proved that the SMARCA5 siRNA interference effect in the experiment was effective.

4. Knockdown of SMARCA5 expression inhibited cell proliferation and invasion in NSCLC

Cell proliferation rate was determined by MTT assay. We found that compared with control siRNA group(Day2-4, $1.56 \pm 0.11$ and $1.50 \pm 0.13, P<0.05, \mathrm{n}=3$ ) or untreated group (Day2-4, $1.71 \pm 0.12$ and $1.45 \pm 0.12, P<0.05, \mathrm{n}=3)$ ), the proliferation rate of A549 and SK-MES-1 decreased significantly after cell transfection with SMARCA5 siRNA 2-4 days(Day2-4, $0.86 \pm 0.05$ and $0.75 \pm 0.06, P<0.01, \mathrm{n}=3$ ) (Fig. 6). These results suggest that silencing SMARCA5 expression can significantly inhibit the proliferation of lung cancer cells.

And transwell test results showed that the number of cells which invaded onto the lower surfaces of the transwell filters in the SMARCA5 siRNA group (A549: $70.64 \pm 5.05$ and SK-MES-1: $85.39 \pm 6.89, P<0.05, \mathrm{n}$ $=3$ ) had less numbers of cells than in the control siRNA group(A549: $256.68 \pm 12.67$ and SK-MES-1: $286.18 \pm 10.64, P<0.05, \mathrm{n}=3$ ), or untreated cells group (A549: $245.41 \pm 10.16$ and SK-MES-1: $300.14 \pm$ 12.17, $P<0.05, \mathrm{n}=3$ ) (Fig. 7). 


\section{Discussion}

The imitation switch nuclear ATPase SMARCA5 (Snf2h) is one of the most conserved chromatin remodeling factors. SMARCA5 expression is upregulated in many aggressive tumors(20-25), several studies have indicated significantly higher expression of SMARCA5 in tumor tissues compared with adjacent normal tissues, including hepatocellular carcinoma (19), gastric cancer(21), and prostate cancer(6). Our research is consistent with the previous reports. We found that SMARCA5 protein expression increased in NSCLC tissues by immunohistochemistry. To further confirm our immunohistochemical observation, we used semi-quantitative RT-PCR and western blot to examine the SMARCA5 expression in paired lung cancer tissues and lung cell lines, and found that SMARCA5 protein and mRNA expression were higher in lung cancer tissues and cells than in nontumorous counterparts.

In addition, we analyzed the relationship between the SMARCA5 expression and the clinical parameters. Then we found that SMARCA5 overexpression significantly associated with advanced TNM stage, pleural invasion and poor differentiation. These results indicated that SMARCA5 upregulated played an important role in lung cancer.

The survival analysis of this study found that the expression of SMARCA5 protein was closely related to the overall survival rate of patients. The overall survival rate of patients with high expression of SMARCA5 were shorter than those with low expression, and the difference in the distribution of survival curves between the two groups was statistically significant. COX multivariate regression analysis also showed that expression of SMARCA5 protein was one of the independent risk factors affecting the prognosis of patients. This indicates that SMARCA5 expression has a certain prognostic effect on NSCLC.

Some study showed that SMARCA5 depletion in breast cancer cells could inhibit invasion with MMP2 downregulation(20). Wang etal reported that overexpression of SMARCA5 in BEL-7402 cells improved the cell growth and knockdown of SMARCA5 expressionin in Huh7 cells inhibited cell growth(19). Others showed that SMARCA5 Deletion Inhibited proliferation of myeloblasts and affected function of normal stem cells(22). Although we showed that SMARCA5 was upregulated in human lung cancer, its biological roles in lung cancer cells was unclear. Then we examined the biological effects of SMARCA5 using SMARCA5 siRNA. We transfedted SMARCA5 siRNA in both A549 and SK-MES-1 cell lines. The results demonstrated that the proliferation capacity in both A549 and SK-MES-1 cell after SMARCA5 knockdown was impaired. We also observed that blocking of SMARCA5 expression inhibited cell invasion. Our results suggest that silencing SMARCA5 probably confers the disadvantage to the growth and invasion of lung cancer cells.

\section{Conclusions}

In conclusion, We found that SMARCA5 expression in lung cancer tissues was significantly upregulated compared with its adjacent normal lung tissues, and was correlated with the clinical features of TNM 
stage, pleural invasion and poor differentiation. The patients with high expression of SMARCA5 had a significant shorter survival. Knockdown of SMARCA5 using RNA interfering showed the decreased activity of proliferation and invasion in NSCLC cells, which indicated that SMARCA5 may play a decisive role in tumorigenesis of NSCLC. Thus, SMARCA5 is a candidate target protein for future lung cancer therapeutics.

\section{Abbreviations}

NSCLC: Non-small cell lung cancer

S-P: streptavidin-peroxidase

DAB: 3, 3'-diaminobenzidine tetrahydrochloride

siRNA: Small interfering RNA

scc: squamous cell carcinoma

\section{Declarations}

\section{Acknowledgements}

Not applicable

\section{Funding}

This work was supported by the Project of Natural Science Foundation of Zhejiang Province (LY16H160058), Medical and Health Science and Technology Project of Zhejiang (2019KY739) and Health Science and Technology Project of Zhoushan City ( 2018G03).

\section{Availability of data and materials}

The datasets used during the current study are available from the corresponding author on reasonable request.

\section{Ethics approval and consent to participate}

This study was approved by the Ethics Committees of Zhoushan Hospital. All lung tissue samples were obtained from zhoushan hospital from January 1, 2007 to December 31, 2016. Written informed consent was obtained from all the participants prior to enrollment.

\section{Consent for publication}

All authors approved for publication of the manuscript for the journal. 


\section{Competing interests}

The authors declare that they have no competing interests.

\section{References}

1 Bray F, Ferlay J, Soerjomataram I, et al. Global cancer statistics 2018:GLOBOCAN estimates of incidence and mortality worldwide for 36 cancers in 185 countries. CA Cancer J Clin. 2018; 68: 394-424.

2 Spicer JF, Rudman SM. EGFR Inhibitors in Non-Small Cell Lung Cancer (NSCLC): The Emerging Role of the Dual Irreversible EGFR/HER2 Inhibitor BIBW 2992. Target Oncol. 2010; 5:245-55.

3 Imbalzano AN, Kwon H, Green MR, et al. Facilitated binding of TATA-binding protein to nucleosomal DNA. Nature. 1994; 370: 481-5.

4 Kwon $\mathrm{H}$, Imbalzano AN, Khavari PA, et al. Nucleosome disruption and enhancement of activator binding by a human SW1/SNF complex. Nature. 1994; 370: 477-81.

5 Wang W, Xue Y, Zhou S, et al. Diversity and specialization of mammalian SWI/SNF complexes. Genes Dev. 1996; 10: 2117-30.

6 Mohamed MA, Greif PA, Diamond J, et al. Epigenetic events, remodelling enzymes and their relationship to chromatin organization in prostatic intraepithelial neoplasia and prostatic adenocarcinoma. BJU Int. 2007; 99: 908-15.

7 LeRoy G, Orphanides G, Lane WS, et al. Requirement of RSF and FACT for transcription of chromatin templates in vitro. Science. 1998; 282: 1900-4.

8 Strohner R, Nemeth A, Jansa P, et al. NoRC-a novel member of mammalian ISWI-containing chromatin remodeling machines. EMBO J. 2001; 20: 4892-900.

9 Bozhenok L, Wade PA, Varga-Weisz P. WSTF-ISWI chromatin remodeling complex targets heterochromatic replication foci. EMBO J. 2002; 21: 2231-41.

10 Banting GS, Barak 0, Ames TM,et al. CECR2, a protein involved in neurulation, forms a novel chromatin remodeling complex with SNF2L. Hum Mol Genet. 2005; 14: 513-24.

11 Stopka T, Skoultchi Al. The ISWI ATPase Snf2h is required for early mouse development. PNAS. 2003; 100: 14097-102.

12 Aydin ÖZ, Vermeulen W, Lans H. ISWI chromatin remodeling complexes in the DNA damage response. Cell Cycle. 2014; 13: 3016-25.

13 Barak O, Lazzaro MA, Lane WS, et al. Isolation of human NURF: a regulator of Engrailed gene expression. EMBO J. 2003; 22: 6089-100. 
14 Lazzaro MA, Pépin D, Pescador N, et al. The imitation switch protein SNF2L regulates steroidogenic acute regulatory protein expression during terminal differentiation of ovarian granulosa cells. Mol Endocrinol. 2006; 20: 2406-17.

15 Song H, Spichiger-Haeusermann C, Basler K. The ISWI-containing NURF complex regulates the output of the canonical Wingless pathway. EMBO Rep. 2009; 10:1140-6.

16 Sala A, Toto M, Pinello L, et al. Genome-wide characterization of chromatin binding and nucleosome spacing activity of the nucleosome remodelling ATPase ISWI. EMBO J. 2011; 30: 1766-77.

17 Wiechens N, Singh V, Gkikopoulos T, et al. The Chromatin Remodelling Enzymes SNF2H and SNF2L Position Nucleosomes adjacent to CTCF and Other Transcription Factors. PLoS Genet. 2016; 12: e1005940.

18 Erdel F, Rippe K. Chromatin remodelling in mammalian cells by ISWI-type complexes-where, when and why? FEBS J. 2011; 278: 3608-18.

19 Wang YN , Qin JX, Liu Q, et al. SNF2H promotes heaptocellular carcinoma proliferation by activating the Wnt/ $\beta$-catenin signaling pathway. Oncol Lett. 2016; 12: 1329-36.

20 Jin QX, Mao XY, Li B, et al. Overexpression of SMARCA5 correlates with cell proliferation and migration in breast cancer. Tumour Biol. 2015; 36:1895-902.

21 Carolina OG, Luara Carolina FL, Mariana FL, et al. SMARCA5 Methylation and Expression in Gastric Cancer. Cancer Invest. 2011; 29: 162-6.

22 Tomas Z, Helena P, Juraj K, et al. Loss of ISWI ATPase SMARCA5 (SNF2H) in Acute Myeloid Leukemia Cells Inhibits Proliferation and Chromatid Cohesion. Int J Mol Sci. 2020; 21: 2073.

23 Sabrina TR, Luciana ST, José PJ, et al. The role of micro RNAs let7c, 100 and 218 expression and their target RAS, C-MYC, BUB1, RB, SMARCA5, LAMB3 and Ki-67 in prostate cancer. Clinics (Sao Paulo). 2013; 68: $652-7$.

24 Jim Jinn-Chyuan S, Jung HC, Isil Y, et al. The roles of human sucrose nonfermenting protein 2 homologue in the tumor-promoting functions of Rsf-1. Cancer Res. 2008; 68: 4050-7.

25 Zhao XC, An P, Wu XY, et al. Overexpression of hSNF2H in glioma promotes cell proliferation, invasion, and chemoresistance through its interaction with Rsf-1. Tumour Biol. 2016; 37: 7203-12.

\section{Figures}



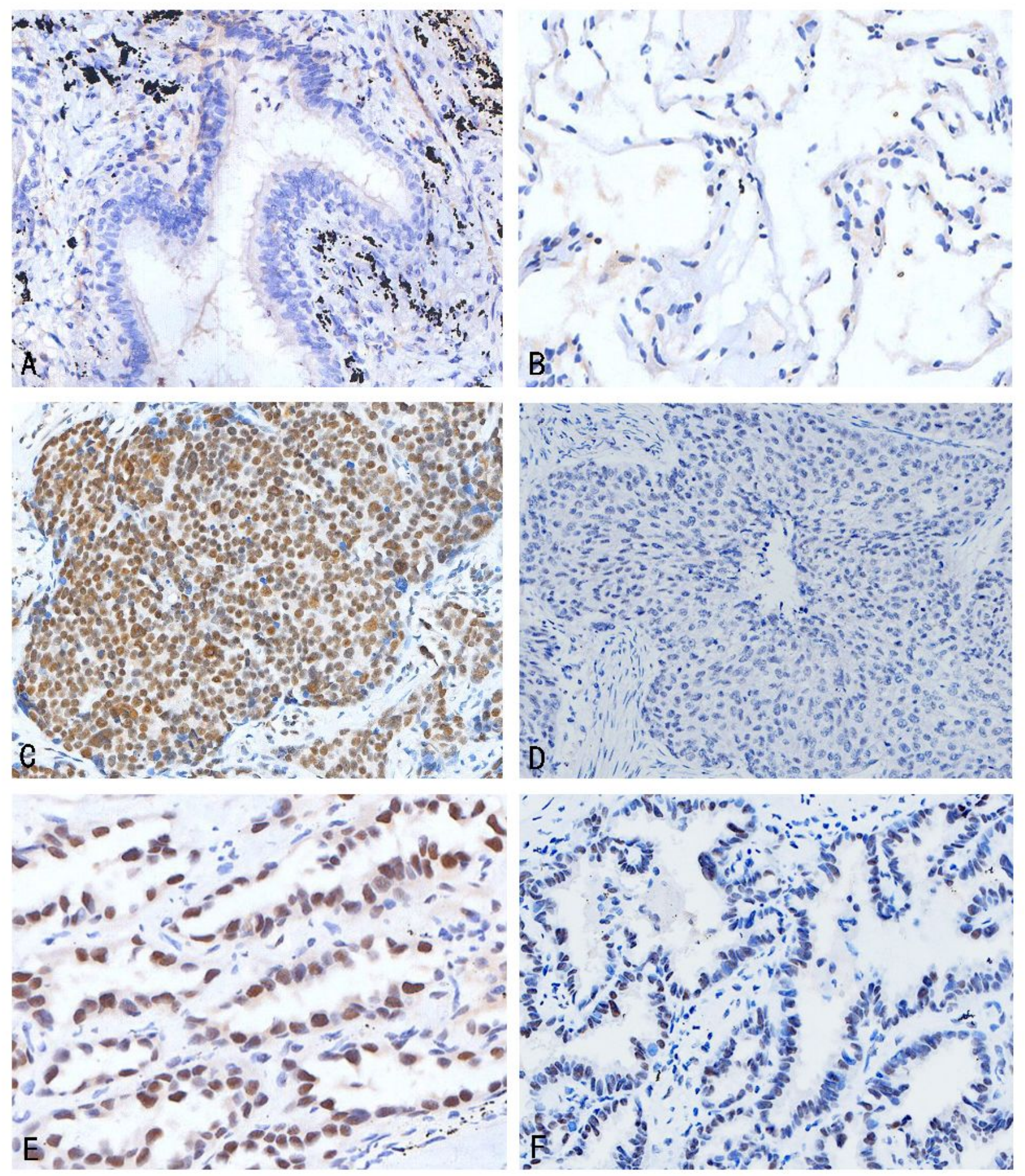

\section{Figure 1}

Expression of SMARCA5 in lung tissues $₫ S-P, \times 200 \rrbracket a, b$ The expression of SMARCA5 protein in normal bronchial epithelium(a) and alveolar epithelium(b) was low or negative. c, $d$ The expression of SMARCA5 protein in lung squamous cell carcinoma(scc) was high(c) or low(d), respectively. e, $f$ The expression of SMARCA5 protein in lung adenocarcinoma was high(e) or low(f), respectively. 
A

$\begin{array}{llllll}\mathrm{N} 1 & \mathrm{~T} 1 & \mathrm{~N} 2 & \mathrm{~T} 2 & \mathrm{~N} 3 & \mathrm{~T} 3\end{array}$

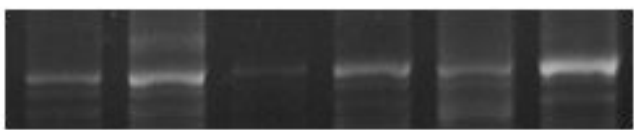

SMARCA5

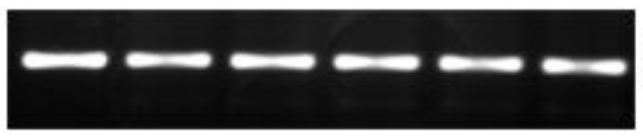

$\beta$-actin

$\mathrm{C}$

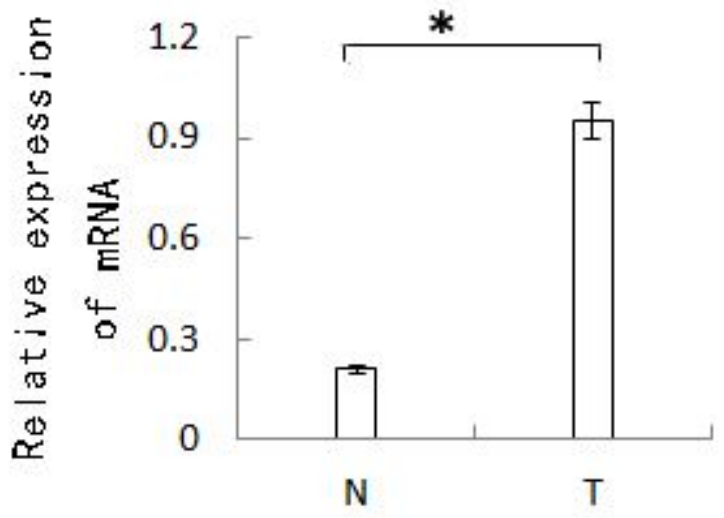

B

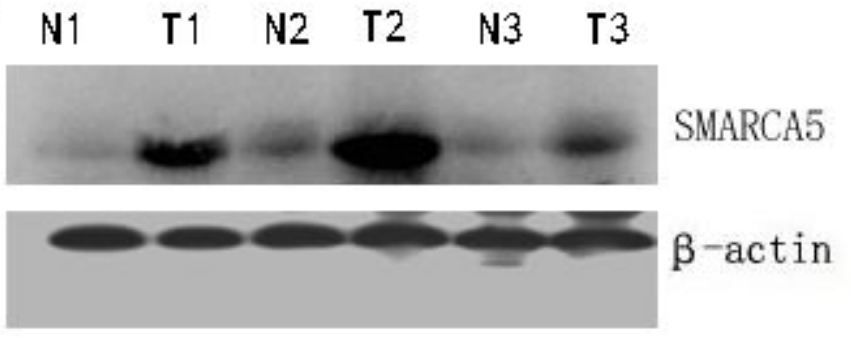

$\mathrm{D}$

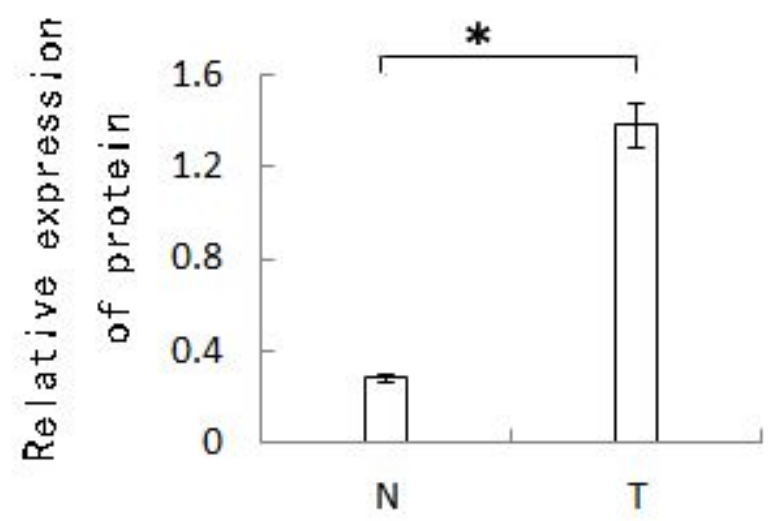

Figure 2

Expression of SMARCA5 in lung cancer tissue and paired adjacent normal tissues $a, b$ The expression of SMARCA5 mRNA and protein in lung cancer tissues was higher than that in paired adjacent normal tissues. $c, d$ The difference of SMARCA5 expression between lung cancer tissues and paired adjacent normal tissues was statistically significant. *, $P<0.05$. Data represented the mean $\pm S D$ of three independent experiments. $\mathrm{N}$, normal tissue $\otimes \mathrm{T}$, tumor tissue. 


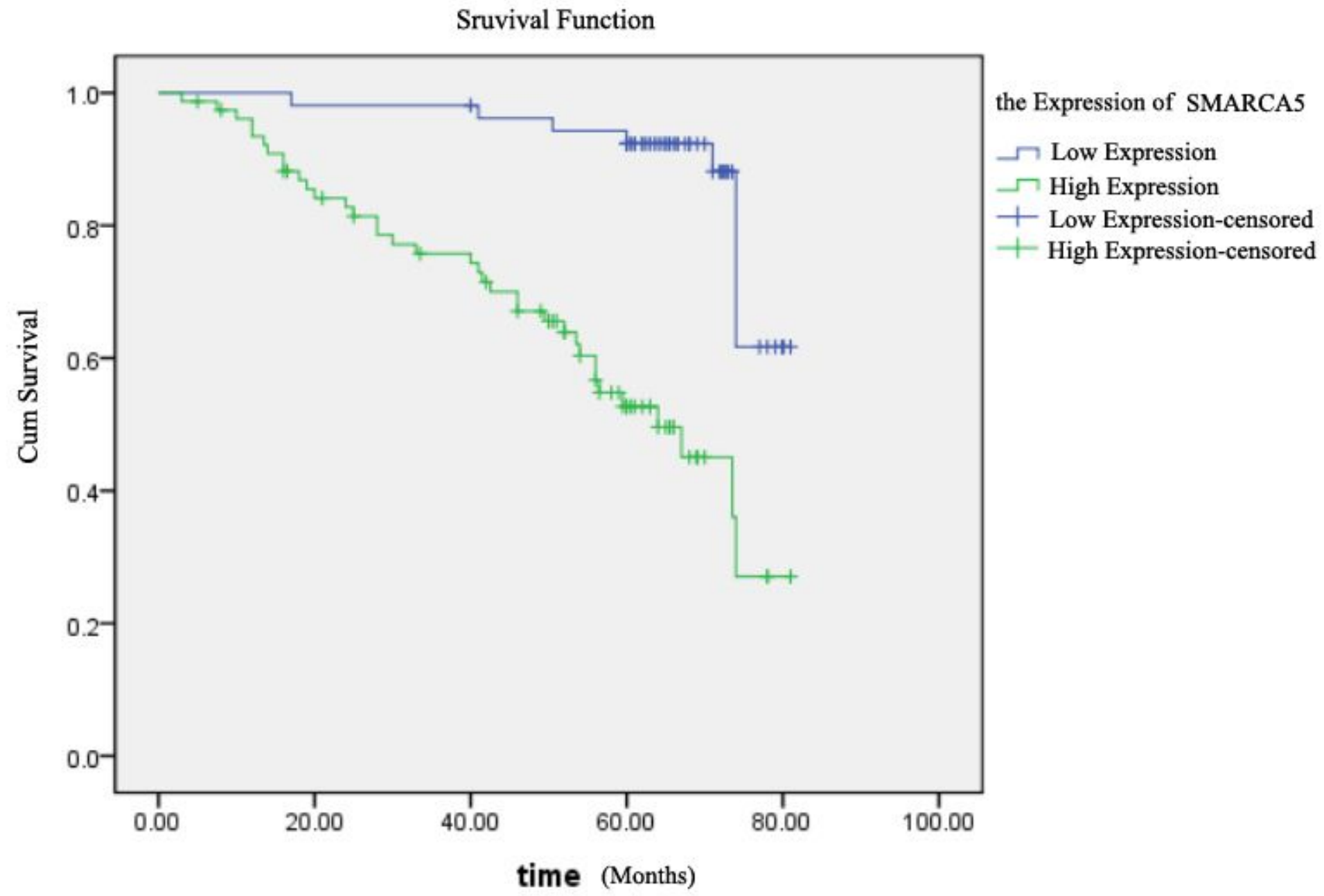

Figure 3

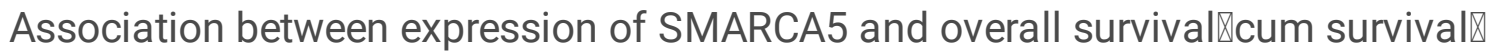


A

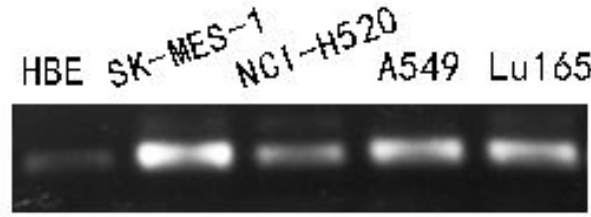

SMARCA5

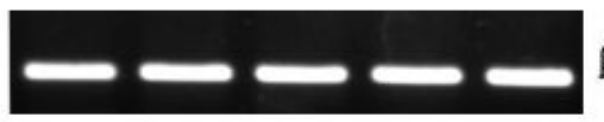

$\beta$-actin

C
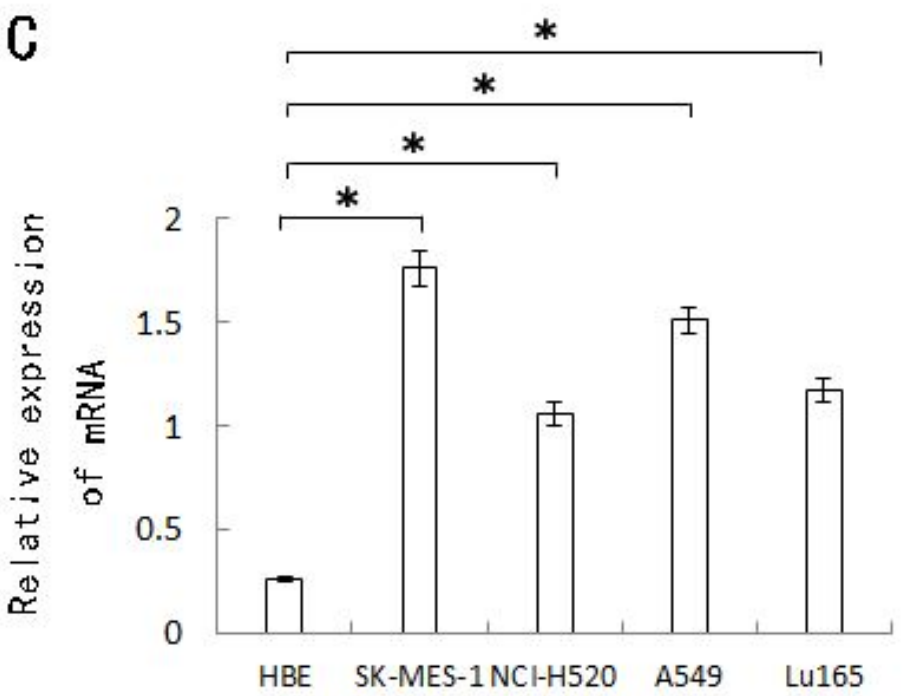

B
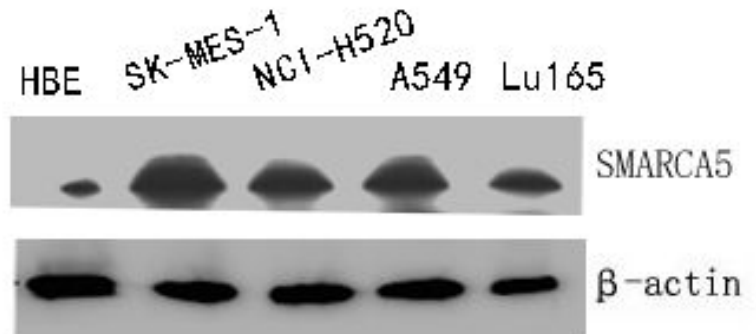

D

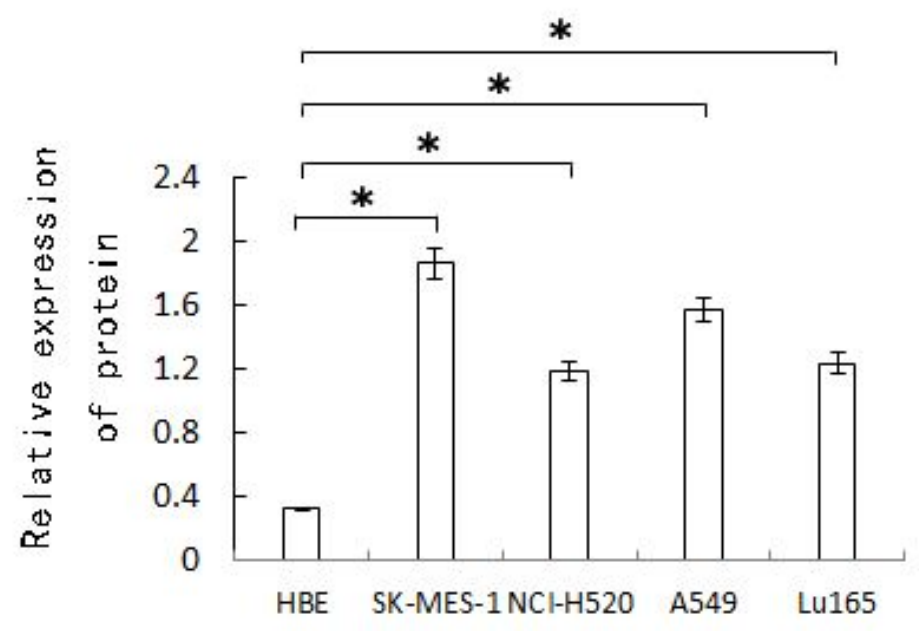

Figure 4

Expression of SMARCA5 in normal bronchial epithelial and lung cancer cell lines $a, b$ The expression of SMARCA5 mRNA and protein in lung cancer cell lines(A549, Lu165, SK-MES-1 and NCI-H520) was higher than that in normal bronchial epithelial cell line区HBEX. $c$, $d$ The expression of SMARCA5 mRNA and protein was analyzed statistically. ${ }^{*}, P<0.05$. Data represented the mean $\pm S D$ of three independent experiments. 

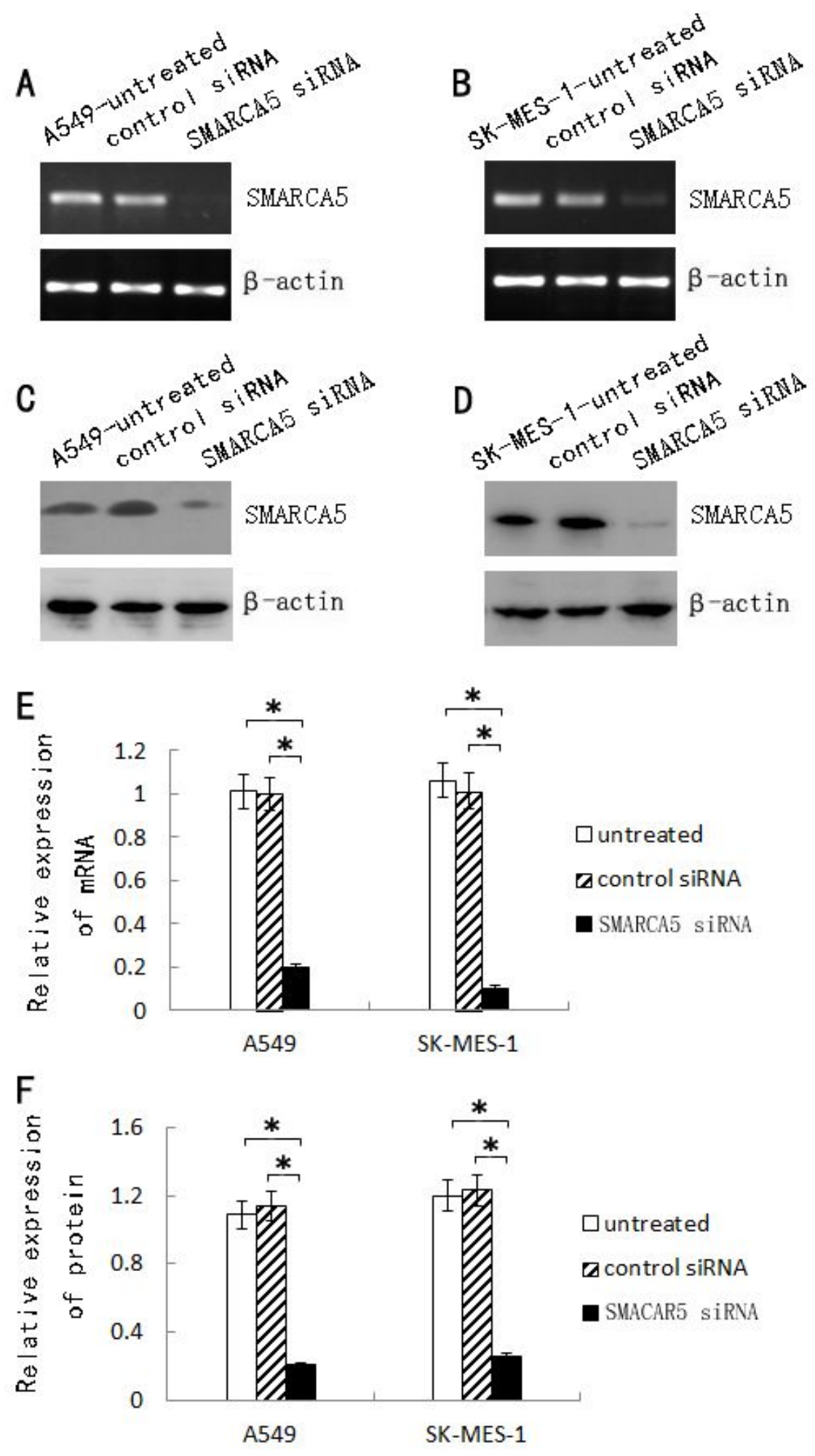

\section{Figure 5}

Silencing SMARCA5 expression in lung cancer cell lines can down-regulate SMARCA5 expression. a-d, RTPCR and Western blot showed that the level of SMARCA5 was decreased inA549 and SK-MES cells transfected with SMARCA5 siRNA compared with the cells transfected with control siRNA or untreated. e, $f$ The expression of SMARCA5 mRNA and protein was analyzed statistically. ${ }^{*}, P<0.05$. Data represented the mean \pm SD of three independent experiments. 

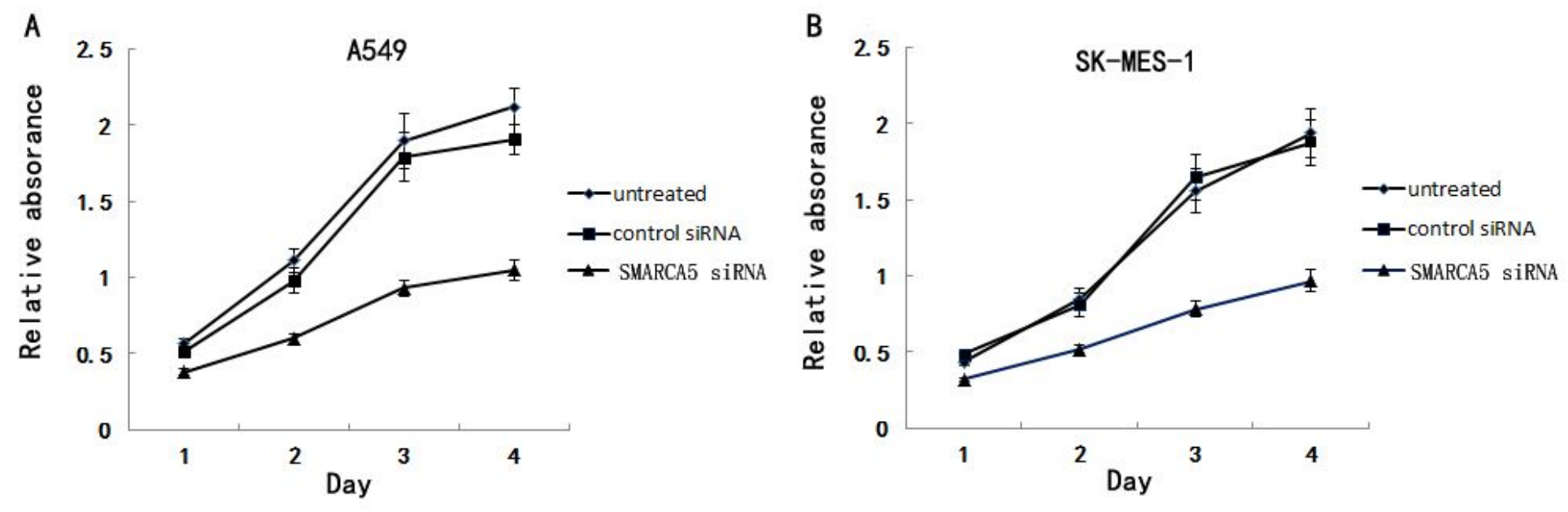

Figure 6

Silencing SMARCA5 expression can inhibit cell proliferation in lung cancer cells 


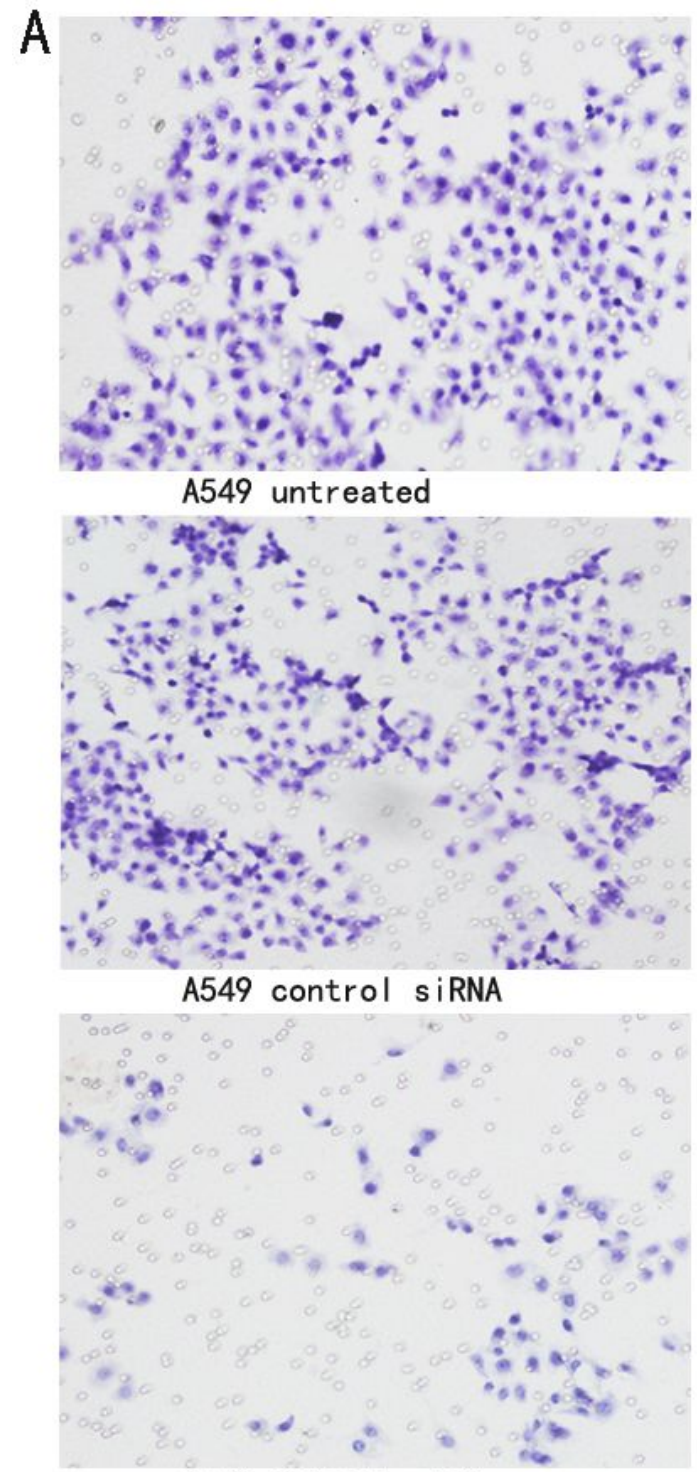

A549 SMARCA5 siRNA

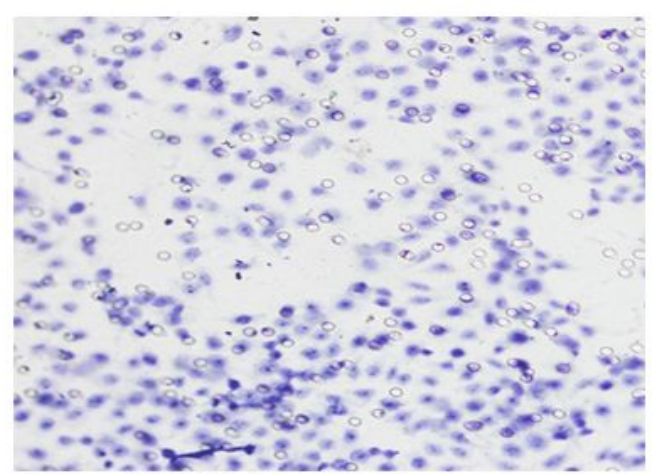

SK-MES-1 untreated

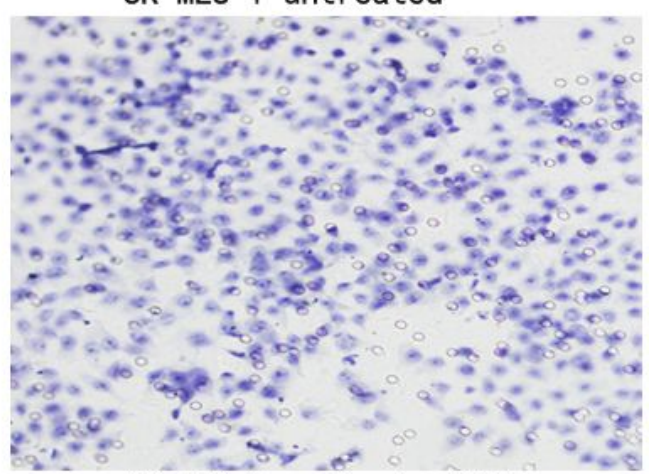

SK-MES-1 control siRNA

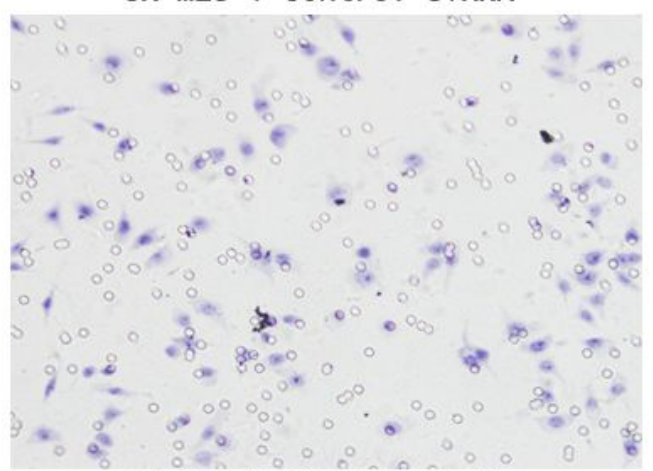

SK-MES-1 SMARCA5 siRNA

B

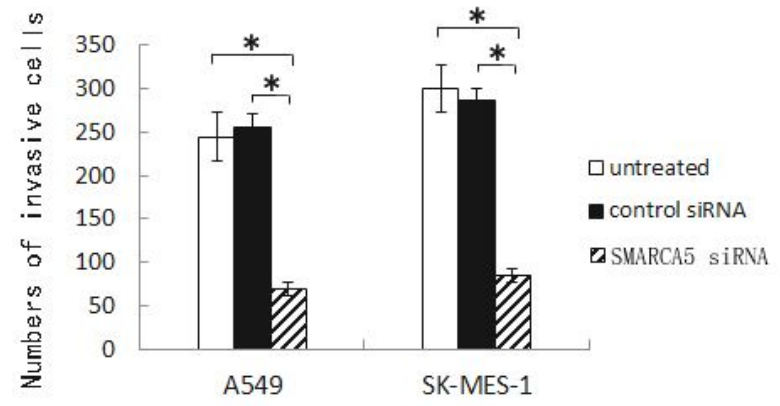

Figure 7

Silencing SMARCA5 expression can inhibit cell invasion in lung cancer cells a, The numbers of cell invasion were decreased inA549 and SK-MES cells transfected with SMARCA5 siRNA compared with the cells transfected with control siRNA or untreated. $b$, The numbers of cell invasion were analyzed statistically. * $P<0.05$. Data represented the mean $\pm S D$ of three independent experiments. 